\title{
Star Formation Thresholds
}

\author{
Joop Schaye ${ }^{1}$ \\ ${ }^{1}$ Leiden Observatory, Leiden University, P.O. Box 9513, 2300 RA Leiden, the Netherlands \\ email: schaye@strw.leidenuniv.nl
}

\begin{abstract}
To make predictions for the existence of "dark galaxies", it is necessary to understand what determines whether a gas cloud will form stars. Star formation thresholds are generally explained in terms of the Toomre criterion for gravitational instability. I contrast this theory with the thermo-gravitational instability hypothesis of Schaye (2004), in which star formation is triggered by the formation of a cold gas phase and which predicts a nearly constant surface density threshold. I argue that although the Toomre analysis is useful for the global stability of disc galaxies, it relies on assumptions that break down in the outer regions, where star formation thresholds are observed. The thermo-gravitational instability hypothesis can account for a number of observed phenomena, some of which were thought to be unrelated to star formation thresholds.
\end{abstract}

Keywords. stars: formation, ISM: structure, galaxies: formation, galaxies: ISM

\section{Introduction}

Do all gas clouds with $M \gg M_{\odot}$ contain stars? And if not, why do some clouds form stars whereas other do not? Observationally, it is nearly impossible to prove that a gas cloud has no stars at all, because a small number of old stars would generally be far too faint to detect. While it is difficult to put upper limits on the amount stellar mass, it is relatively easy to obtain interesting upper limits on the amount of star formation. This can for example be done through the non-detection of recombination lines such as $\mathrm{H} \alpha$, which are generated in the HII regions around young stars, or through infrared observations.

The H I components of most spiral galaxies are observed to extend beyond the region of active star formation. It has been known for some time, that star formation is generally limited to regions where the total gas surface density exceeds $\Sigma_{\mathrm{c}} \sim 10 \mathrm{M}_{\odot} \mathrm{pc}^{-2}$ (e.g., Guiderdoni 1987; Skillman 1987, Taylor et al. 1994). The existence of a surface density threshold for star formation is, however, usually explained in terms of the Toomre criterion for gravitational instability (e.g., Quirk 1972; Kennicutt 1989). Neither rotation nor pressure can stabilize a thin, gaseous, differentially rotating disk if the Toomre $Q$ parameter,

$$
Q \equiv \frac{c_{\mathrm{s}} \kappa}{\pi G \Sigma_{\mathrm{g}}}
$$

is less than unity (Safronov 1960; Toomre 1964); where the effective sound speed $c_{\mathrm{s}}$, the epicycle frequency $\kappa$, and the gas surface density $\Sigma_{\mathrm{g}}$ all depend on radius $r$. According to this theory, star formation is suppressed when the gas surface density falls below the critical value

$$
\Sigma_{\mathrm{c}} \equiv \frac{c_{\mathrm{s}} \kappa}{\pi G} \equiv Q \Sigma_{\mathrm{g}}
$$

which depends on the local values of the velocity dispersion and the epicycle frequency.

Kennicutt (1989) and Martin \& Kennicutt (2001, hereafter MK01) tested the hypothesis that the Toomre criterion is responsible for the observed cutoff in the star formation 
rate by measuring the ratio $\alpha \equiv 1 / Q$ of the gas surface density $\Sigma_{\mathrm{g}}$ to the critical surface density. Assuming a constant velocity dispersion of $6 \mathrm{~km} \mathrm{~s}^{-1}$ and after azimuthal averaging, MK01 found that $\alpha \equiv \Sigma_{\mathrm{g}} / \Sigma_{\mathrm{c}} \approx 0.5 \dagger$ at the truncation radius in a sample of 32 nearby spiral galaxies.

Elmegreen \& Parravano (1994) emphasized that the need for a cold gas phase imposes a pressure threshold. Schaye (2004, hereafter S04) showed that the transition from the warm to the cold gas phase triggers gravitational instability on a wide range of scales. He demonstrated that this thermo-gravitational instability can account for the observed star formation threshold, which he predicted to fall in the range $\Sigma_{\mathrm{c}} \approx 3-10 \mathrm{M}_{\odot} \mathrm{pc}^{-2}$ (corresponding to a critical pressure $P_{c} / k \sim 10^{2}-10^{3} \mathrm{~cm}^{-3} \mathrm{~K}$ and a critical volume density $n_{\mathrm{H}} \sim 10^{-2}-10^{-1} \mathrm{~cm}^{-3}$ ), with weak dependencies on the metallicity, UV radiation field, turbulent pressure, and the mass fraction in gas.

After briefly summarizing the S04 model in section 2, I argue in section 3 that the Toomre $Q$ hypothesis relies on assumptions that break down in the outer parts of galaxies, where star formation thresholds are observed. In section 4 I discuss a number of observational findings that are naturally explained by the thermo-gravitational instability hypothesis.

In the second part of my talk, I discussed the relation between star formation laws expressed in terms of surface densities, volume densities and pressures. I presented a method based on this theory with which it is possible to reproduce arbitrary KennicuttSchmidt laws using numerical simulations, without any free parameters. I do not have the space here to describe this model and its implications for the interpretation of simulations. For more on this topic, stay tuned for the paper "On the relation between the Schmidt and Kennicutt-Schmidt star formation laws and its implication for numerical simulations" (Schaye \& Dalla Vecchia, in preparation).

\section{Thermo-gravitational instability}

The insterstellar medium of galaxies is highly complex. It contains a number of gas phases, including a cold $\left(T \lesssim 10^{2} \mathrm{~K}\right)$, warm $\left(T \sim 10^{4} \mathrm{~K}\right)$, and hot $\left(T \gg 10^{4} \mathrm{~K}\right)$ phase. Feedback from star formation drives strong, supersonic turbulence which can both promote and suppress gravitational stability. Because of this complexity, it is difficult to predict exactly where and when star formation occurs. However, the outer parts of galaxies are much simpler. Here the gas is almost exclusively warm and there is no, or very little, star formation and hence turbulence is weak and typically subsonic. Moving in towards the center, we may therefore be able to predict where star formation begins, even if we cannot predict what happens further in.

S04 noted that the UV background radiation implies the existence of a threshold surface density for the formation of a cold phase, which agrees with the empirically determined star formation threshold. He hypothesized that the drop in the pressure associated with the transition to a cold phase triggers gravitational instability and thus star formation. Conversely, for densities below the threshold, self-gravitating gas clouds are kept warm and stable by the UV radiation.

To test this hypothesis, S04 constructed two model galaxies consisting of exponential discs embedded in cold dark matter halos. The discs were illuminated by UV radiation and contained metals and dust. For a fixed surface density, the temperature and volume density were determined numerically under the condition of vertical hydrostatic

$\dagger$ We corrected their value of $\alpha$, see Schaye (2004). 

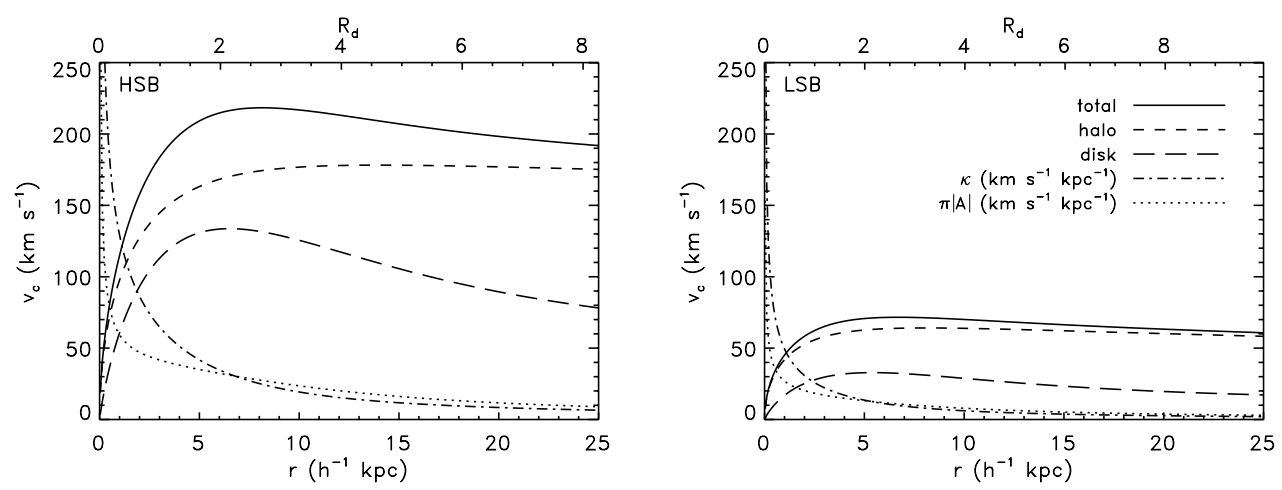

Figure 1. Circular velocity as a function of radius for models HSB (left) and LSB (right). The short (long) dashed curve shows the contributions of the halo (disc). Also shown are the epicycle frequency (dot-dashed curves) and $\pi$ times Oort's constant $A$ (dotted curves), both in $\mathrm{km} \mathrm{s}^{-1} \mathrm{kpc}^{-1}$. Figure taken from S04.
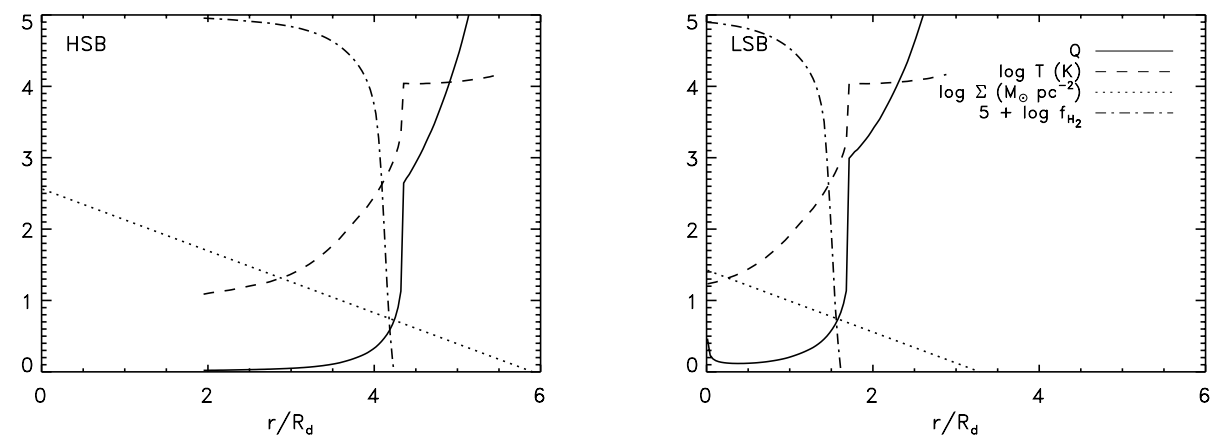

Figure 2. The Toomre $Q$ parameter (solid curves), the temperature $\log T$ (dashed curves), the molecular fraction $5+\log f_{H_{2}}$ (dot-dashed curves) and the surface density $\log \Sigma\left(\mathrm{M}_{\odot} \mathrm{pc}^{-2}\right)$ (dotted curves) are all plotted as a function of radius for models HSB (left) and LSB (right). Figure taken from S04.

equilibrium. The radiative transfer and chemistry were calculated using the publicly available package CLOUDY (Ferland 2000).

The parameters of the model galaxies were chosen to mimic a high and a low surface brightness galaxy. Figure 1 shows the rotation curves (solid), including the contributions of the halo (short dashed) and disc (long dashed), the epicycle frequency (dot-dashed) and $\pi$ times Oort's constant (dotted).

The solid curves in Figure 2 show the Toomre $Q$ parameter as a function of radius. The sudden drop in the $Q$ values coincides with (and is caused by) a similar drop in the temperature (dashed curves), and a sharp increase in the molecular fraction (dot-dashed curves). The transition to the cold phase, which coincides with the onset of gravitational instability $(Q<1)$, occurs at a fixed surface density. Note that the models become unrealistic shortwards of the critical radius, where feedback from star formation will increase the UV field, the metallicity, and the turbulent pressure.

Figure 3 again shows the $Q$ parameter (thick solid curves) as a function of radius, but note that this time the $y$-axis is logarithmic. The value $Q=1$ is indicated with solid, horizontal lines. A Toomre $Q$ of unity implies marginal instability only for fluctuations 

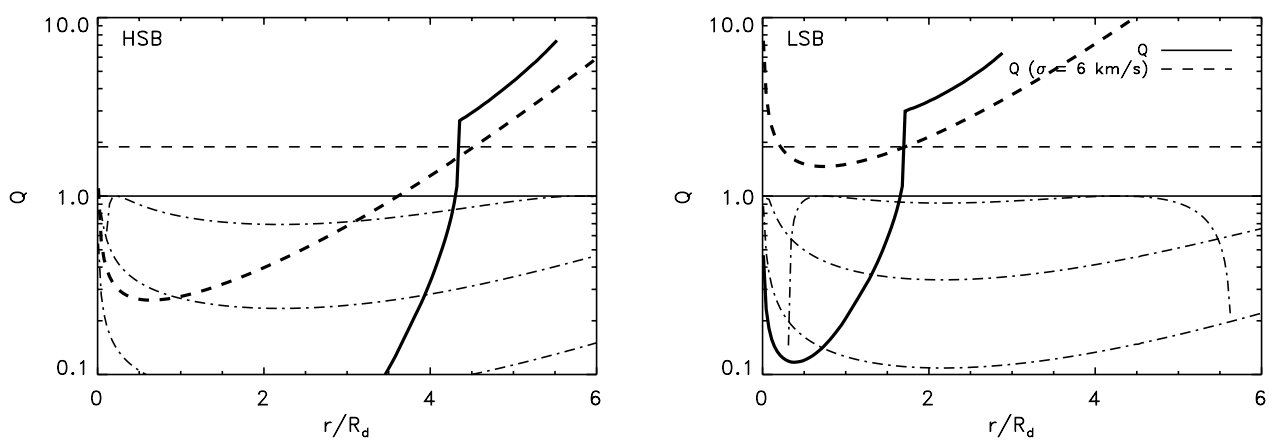

Figure 3. The Toomre $Q$ parameter as a function of radius (thick solid curves) for models HSB (left) and LSB (right). The solid horizontal lines indicate $Q=1$. The dot-dashed curves indicate the $Q$ values below which the disc is unstable to perturbations with wavelengths of (top) $10^{3}$, (middle) $10^{2}$, and (bottom) 10 pc. The thick dashed curves show the Toomre $Q$ parameter computed under the assumption that $\sigma=6 \mathrm{~km} \mathrm{~s}^{-1}$. The horizontal dashed lines indicate the critical $Q$ value measured by Kennicutt (1989) under the assumption that $\sigma=6 \mathrm{~km} \mathrm{~s}^{-1}$. Figure taken from S04.

with $\lambda=\lambda_{\text {crit }} / 2$, where

$$
\lambda_{\text {crit }} \equiv \frac{4 \pi^{2} G \Sigma_{\mathrm{g}}}{\kappa^{2}}
$$

In general, instability of perturbations on scale $\lambda$ requires $Q<Q_{c}(\lambda)$, where

$$
Q_{c}(\lambda)=2 \sqrt{\frac{\lambda}{\lambda_{\text {crit }}}-\left(\frac{\lambda}{\lambda_{\text {crit }}}\right)^{2}}
$$

The dot-dashed curves in Figure 3 indicate the $Q$ values below which the disc is unstable to perturbations with wavelengths of (top) $10^{3}$, (middle) $10^{2}$, and (bottom) 10 pc. The transition to the cold phase causes $Q$ to drop far below unity, thereby triggering instability on a wide range of scales.

Assuming a constant velocity dispersion of $\sigma=6 \mathrm{~km} \mathrm{~s}^{-1}$ (thick dashed curves) would result in large errors in the $Q$-parameter. However, if we recalibrate the critical $Q$ parameter and compare with the value measured by Kennicutt (1989) (horizontal dashed lines), then we obtain agreement between the predicted critical radii (the intersections of the thick solid curves and the horizontal solid lines roughly coincide with the intersections of the thick dashed curves and the horizontal dashed lines).

S04 computed a large range of models and found that the following function can describe the variation of the critical surface density with the parameters of the model

$$
\log \left(\frac{\Sigma_{\mathrm{c}}}{1 \mathrm{M}_{\odot} \mathrm{pc}^{-2}}\right) \approx 0.8+0.3 \log (f)-0.3 \log \left(\frac{Z}{0.1 Z_{\odot}}\right)+0.2 \log \left(\frac{I}{10^{6} \mathrm{~cm}^{-2} \mathrm{~s}^{-1}}\right)
$$

where $Z$ is the metallicity, $I$ is the flux of ionizing radiation, and $f \equiv f_{g} / f_{\text {th }}$, with $f_{\mathrm{g}}$ the mass fraction in gas and $f_{\text {th }}$ the fraction of the pressure that is thermal. The above formula (see S04 for second order corrections) was found to reproduce the results for a wide variety of galaxy models. 


\section{The assumptions underlying the Toomre hypothesis are invalid in outer discs}

In this section I will argue that the Toomre analysis is generally not applicable to star formation thresholds in the outer parts of disc galaxies, because the underlying assumptions are invalid. Below I will discuss the offending assumptions in turn.

A Toomre $Q$ smaller than unity is sufficient for star formation. A Toomre $Q$ of unity implies marginal instability only for fluctuations with $\lambda=\lambda_{\text {crit }} / 2$. In the outer parts of disc galaxies, this critical wavelength is typically $>1 \mathrm{kpc}$ (see Fig. 3). Hence, while $Q=1$ is relevant for large-scale modes such as spiral arms, we require fluctuations with much smaller wavelengths to become unstable to account for the formation of star clusters. Note that if $\Sigma_{\mathrm{g}} \sim \Sigma_{\mathrm{c}}\left(\sigma=6 \mathrm{~km} \mathrm{~s}^{-1}\right)$ is observed, then the condition $Q \ll 1$ implies $\Sigma_{\mathrm{c}} \ll \Sigma_{\mathrm{c}}\left(\sigma=6 \mathrm{~km} \mathrm{~s}^{-1}\right)$ and thus $\sigma \ll 6 \mathrm{~km} \mathrm{~s}^{-1}$.

The disc is thin. In the outer parts of galaxies, the disc scale height is similar to the most unstable wavelength, thereby invalidating the assumption that the disc is thin.

The perturbations are axisymmetric. Beyond the truncation radius identified in azimuthally averaged observations, star formation does occur in isolated regions whenever the local gas surface density exceeds the global threshold (e.g., Ferguson et al. 1998). The Toomre analysis is valid only for axisymmetric perturbations and can therefore not straightforwardly be applied to such regions.

The velocity dispersion is constant. Although the Toomre analysis does not require it, when it is applied to star formation thresholds it is generally assumed that the velocity dispersion is constant. Following Kennicutt (1989), most work assumes $\sigma=6 \mathrm{kms}^{-1}$. For sufficiently large scale heights, the gas will be kept warm by the UV background radiation. Therefore, there will always be a component with a velocity dispersion of at least that of the warm phase. However, if there is any cold gas then the relevant velocity dispersion, and thus $Q$, can locally be much smaller.

Rotation needs to be taken into account. As mentioned above, to form stars, or even star clusters, we require instability for $\lambda \ll \lambda_{\text {crit }}$ and hence $Q \ll 1$. However, for $\lambda \ll \lambda_{\text {crit }}$ the Toomre criterion reduces to the two-dimensional Jeans criterion: $\lambda>$ $c_{\mathrm{s}}^{2} / G \Sigma_{\mathrm{g}}$. Hence, rotation is generally not directly relevant for star formation thresholds. The exception is the central part of the disc, where $\lambda_{\text {crit }} \propto \kappa^{-2}$ becomes small (see Fig. 1) and the Coriolis force can, in principle, prevent collapse on scales relevant for star formation. However, in practice the disc cannot be considered thin in the central parts because the scale height is no longer small compared with the radius. Moreover, the bulge will need to be taken into account.

\section{Observations that are naturally explained by the thermo-gravitational instability}

A number of observational findings, some of which were thought to be unrelated, can be explained by the thermo-gravitational instability theory of star formation thresholds. Below I will discuss the most important ones.

\subsection{Why is the critical surface density constant?}

Why can a single constant, surface density threshold account for the observed star formation thresholds (e.g., Guiderdoni 1987; Skillman 1987, Taylor et al. 1994)? A constant threshold works works even on a pixel-by-pixel basis for high-resolution observations of nearby galaxies (de Blok \& Walter 2006) and it can also account for the isolated star formation events beyond the optical radii of galaxies (e.g., Ferguson et al. 1998). 
If the Toomre hypothesis holds, then $\Sigma_{\mathrm{c}} \propto c_{\mathrm{s}} \kappa$ and should therefore vary from place to place. Even if we fix $c_{\mathrm{s}}$, as is often done, it should still depend on the epicycle frequency. If the thermo-gravitational instability is responsible, then the critical surface density will be nearly constant [see equation (2.3)] and the predicted value is in excellent agreement with the observations.

Note that although $\Sigma_{\mathrm{c}}$ is predicted to depend only very weakly on environmental factors such as the metallicity and the UV intensity, the differences may still become noticeable in extreme environments. For example, we predict $\Sigma_{\mathrm{c}} \gg 10 \mathrm{M}_{\odot} \mathrm{pc}^{-2}$ for the first generation of stars at very high redshift (because $Z \ll 0.1 Z_{\odot}$ ) and also very near strong sources of UV radiation.

\subsection{Why is a large fraction of disc galaxies inconsistent with the Toomre hypothesis?}

Why does a constant threshold work even when the Toomre criterion does not? Many studies have found so-called "subcritical discs". That is, galaxies for which $Q(\sigma=$ $6 \mathrm{~km} \mathrm{~s}^{-1}$ ) exceeds the critical value throughout the region of active star formation (e.g., MK01; Wong \& Blitz 2002; Auld et al. 2006). About 1 in 4 of the galaxies from the sample of MK01 fall in this category. As can be seen from the figures in these papers, the subcritical discs do agree with the constant star formation threshold hypothesis.

\subsection{Why is the velocity dispersion in the outer disc $8 \mathrm{~km} \mathrm{~s}^{-1}$ ?}

Why is the velocity dispersion of the outer H I disk constant and why does it have the value $8 \mathrm{~km} \mathrm{~s}^{-1}$ (e.g., Lo et al. 1993; Meurer et al. 1996)? Neither magnetically induced (Sellwood \& Balbus 1999) nor gravitationally induced (e.g., Wada et al. 2002) turbulence can account for such a high (and constant) value.

According to the thermo-gravitational instability picture, the gas in the outer disk, at surface densities below the threshold, is kept warm by the UV background radiation. Although this should be uncontroversial, theoretical studies have rarely taken radiation into account.

\subsection{Why does the same $\Sigma_{\mathrm{c}}$ also work for tidal arms?}

Maybhate et al. (2007) found that the standard, constant surface density threshold can also account for observations of star clusters in tidal arms. Tidal arms can hardly be considered part of thin, differentially rotating discs, and the Toomre hypothesis is therefore even less applicable to these observations than to those in the outer parts of disc galaxies. However, the thermo-gravitational instability does not assume a thin, rotating disc and should thus hold equally well in tidal arms.

\subsection{Why is gas with $\Sigma_{\mathrm{g}}<\Sigma_{\mathrm{c}}$ nearly always warm?}

Why does the contribution of the cold interstellar phase to the gas surface density become negligible beyond the edge of the star forming disk (Braun 1997)? This observation is in excellent agreement with the predictions of the thermo-gravitational instability hypothesis: the formation of a cold phase triggers star formation and the two therefore go hand in hand. For $\Sigma<\Sigma_{\mathrm{c}}$ the cold gas fraction, and thus the star formation rate, plummets.

In the constant velocity dispersion version of the Toomre hypothesis there is no 1-1 relationship between the ability to form a cold phase and the ability to form stars. While instability of the warm phase will presumably also eventually trigger the formation of a cold phase, the converse does not need to be true. Therefore, there is no reason why there could not be lots of cold gas in regions where there is no star formation. 
4.6. Why does the H I surface density saturate at $\Sigma_{\mathrm{HI}} \sim \Sigma_{\mathrm{c}}$

Many observations indicate that while the H I surface density increases with decreasing galactocentric radius, it typically saturates around $10 \mathrm{M}_{\odot} \mathrm{pc}^{-2}$ (e.g., Wong \& Blitz 2002). At smaller radii the total gas surface density can be much higher, but for $\Sigma_{\mathrm{g}} \gg$ $10 \mathrm{M}_{\odot} \mathrm{pc}^{-2}$ most of the gas is molecular. Is it a coincidence that the H I surface density saturates at the star formation threshold? If the Toomre hypothesis is correct, then it would be. Although star formation may destroy the H I, there will be galaxies for which the disc is Toomre stable for $\Sigma_{\mathrm{g}} \gg 10 \mathrm{M}_{\odot} \mathrm{pc}^{-2}$.

In Schaye (2001) I pointed out that there is a physical upper limit to the H I surface density of gas clouds because the gas will quickly go molecular beyond a critical density, which depends weakly on the metallicity and the UV radiation field. The transition to a cold, molecular phase both limits the H I surface density and triggers star formation. Thus, the agreement between the critical surface density for star formation and the H I saturation limit is a prediction of the thermo-gravitational instability picture.

It has been argued that MK01's finding that the molecular fraction varies wildly at the critical radius for star formation is evidence against the thermo-gravitational hypothesis. However, a large scatter in the molecular fraction is exactly what is expected here. The critical radius $R_{c}$ is defined (by eye) as the radius within which star formation is ubiquitous and beyond which it is sporadic. The prediction is that for $R \gg R_{c}$ the gas is mostly atomic, while for $R \ll R_{c}$ it is mostly molecular (provided that $\Sigma_{\mathrm{g}}(r)$ is well behaved so that $\Sigma_{\mathrm{g}} \ll \Sigma_{\mathrm{c}}$ for $R \gg R_{c}$ and $\Sigma_{\mathrm{g}} \gg \Sigma_{\mathrm{c}}$ for $R \ll R_{c}$ ). Since $R \sim R_{c}$ is the transition regime, we expect both atomic and molecular regions. Azimuthal averaging will then result in a wide range of molecular fractions.

\subsection{Why is the empirically determined critical $Q$ different for irregulars?}

MK01 find that star formation is suppressed when $\Sigma_{\mathrm{g}} / \Sigma_{\mathrm{c}}>\alpha=0.5$. On the other hand, Hunter, Elmegreen, \& Baker (1998) measured a smaller value of $\alpha$ for a sample of irregulars. However, Hunter et al. (1998) used a velocity dispersion of $9 \mathrm{~km} \mathrm{~s}^{-1}$ because the standard value of $6 \mathrm{~km} \mathrm{~s}^{-1}$ was inconsistent with the observations. Had they assumed used $6 \mathrm{~km} \mathrm{~s}^{-1}$, they would have inferred a value of $\alpha$ that agrees within $1 \sigma$ with the Kennicutt (1989) value. This again reflects the fact that a constant surface density threshold describes the observations very well. If $\sigma$ is increased, then a constant surface density threshold implies a corresponding reduction of $\alpha$.

\section{Conclusions}

S04 argued that star formation thresholds are set by the necessity to form a cold gas phase. Below a critical surface density the gas is kept warm by UV radiation. Conversely, when the surface density exceeds the threshold, the drop in the gas pressure associated with the phase transition triggers gravitational instability. As we discussed above, this hypothesis is in excellent, quantitative agreement with observations of the outer parts of galaxies. It can account for the observed star formation thresholds and the observed velocity dispersion, it can explain the lack of cold gas beyond regions of active star formation, and it also explains the coincidence between the critical surface density and the H I saturation limit.

Does the Toomre $Q$ play any role at all? It almost certainly does play an important role. Although it is not applicable to the outer parts, the molecular disc is thin compared with $\lambda_{\text {crit }}$ and can thus be characterized using the Toomre parameter. If $Q<1$ then the disc is unstable to large-scale axisymmetric perturbations, which will for example lead to the formation of spiral arms. The resulting increase in the surface density can 
trigger local instabilities, and not necessarily only through the formation of a cold phase. However, on scales relevant to the formation of star clusters, the 3-D Jeans criterion describes the local stability better than the local Toomre $Q$, and much better than the constant velocity dispersion version of the Toomre criterion.

\section{Acknowledgements}

I would like to thank the organizers for what turned out to be a very stimulating conference. This work was supported by Marie Curie Excellence Grant MEXT-CT-2004014112 .

\section{References}

Auld, R., de Blok, W. J. G., Bell, E. \& Davies, J. I. 2006, MNRAS, 366, 1475

Braun, R. 1997, ApJ, 484, 637

de Blok, W. J. G. \& Walter, F., 2006, AJ, 131, 363

Elmegreen, B. G. \& Parravano, A. 1994, ApJ, 435, L121

Ferguson, A. M. N., Wyse, R. F. G., Gallagher, J. S. \& Hunter, D. A. 1998, ApJ, 506, L19

Ferland, G. J. 2000, Revista Mexicana de Astronomia y Astrofisica Conference Series, 9, 153

Guiderdoni, B., 1987, A\&A, 172, 27

Hunter, D. A., Elmegreen, B. G. \& Baker, A. L. 1998, ApJ, 493, 595

Kennicutt, R. C. 1989, ApJ, 344, 685

Lo, K. Y., Sargent, W. L. W. \& Young, K. 1993, AJ, 106, 507

Martin, C. L. \& Kennicutt, R. C. 2001, ApJ, 555, 301 (MK01)

Maybhate A., Masiero J., Hibbard J. E., Charlton J. C., Palma C., Knierman K. A. \& English J., 2007, arXiv, 707, arXiv:0707.3582

Meurer, G. R., Carignan, C., Beaulieu, S. F. \& Freeman, K. C. 1996, AJ, 111, 1551

Quirk, W. J. 1972, ApJ, 176, L9

Schaye, J. 2001, ApJ, 562, L95

Schaye, J. 2004, ApJ, 609, 667 (S04)

Sellwood, J. A. \& Balbus, S. A. 1999, ApJ, 511, 660

Skillman, E. D. 1987, in Star Formation in Galaxies, edited by C. J. Londsdale Persson (NASA Conf. Pub. CP-2466), p. 263

Taylor, C. L., Brinks, E., Pogge, R. W. \& Skillman, E. D. 1994, AJ, 107, 971

Wada, K., Meurer, G. \& Norman, C. A. 2002, ApJ, 577, 197

Wong, T. \& Blitz, L. 2002, ApJ, 569, 157

\section{Discussion}

TAYLOR: Recent GALEX observations do not show evidence for star formation thresholds. How do you interpret those observations?

SCHAYE: The GALEX observations are very interesting. At first sight they appear to contradict the $\mathrm{H} \alpha$ observations, which do show evidence for star formation thresholds. GALEX detects star light, whereas the $\mathrm{H} \alpha$ comes from $\mathrm{H}$ II regions. GALEX is sensitive to star formation up to $100 \mathrm{Myr}$ after the stars formed, whereas the $\mathrm{H} \alpha$ becomes difficult to detect after $10 \mathrm{Myr}$. The latter observations therefore offer more direct tests of theories of star formation thresholds. It is difficult to use GALEX observations to relate star formation to local gas densities because after 100 Myr feedback from star formation may well have destroyed/removed all the local $\mathrm{H} \mathrm{I}$ and $\mathrm{H}_{2}$. GALEX may probe the cumulative effect of the isolated star formation events that are seen in $\mathrm{H} \alpha$ in the outer discs. 
TAYLOR: Stellar discs may not be truncated, although many do show evidence for a change in the exponential scale length in the outer parts. Can star formation thresholds explain this?

SCHAYE: Star formation does occur in the far outer parts of galaxies, when the local gas density exceeds the threshold. I could imagine some kind of change in the stellar surface density profile around the point where the azimuthally averaged gas surface density equals the threshold. Within that critical radius the star formation rate will be mostly determined by the efficiency of star formation rather than by the threshold. On the other hand, beyond the critical radius it will be determined more by the frequency of density fluctuations strong enough to exceed the threshold. However, the stellar disc is built up over long time scales, during which the critical radius may vary. Moreover, stars may move radially after they formed and stars in the outer discs may also have been accreted. These effects complicate the relationship between star formation thresholds and stellar surface density profiles. 